\title{
Salivary HOTAIR and PVT1 as novel biomarkers for early pancreatic cancer
}

\author{
Zijun Xie ${ }^{1, *}$, Xiaoliang Chen ${ }^{1, *}$, Jianzhong $\mathrm{Li}^{1, *}$, Yunwei Guo $^{1}$, Haijiao $\mathrm{Li}^{1}$, Xuemei Pan ${ }^{1}$, \\ Jie Jiang ${ }^{1}$, Huiling Liu ${ }^{1}$, Bin W $\mathbf{u}^{1}$ \\ ${ }^{1}$ Department of Gastroenterology, The Third Affiliated Hospital, Sun Yat-Sen University, Guangzhou, China \\ *These authors contributed equally to this work
}

Correspondence to: Bin Wu, e-mail: wubin6@mail.sysu.edu.cn

Keywords: HOTAIR, PVT1, pancreatic cancer, biomarker, diagnosis

Received: December 16, 2015

Accepted: March 07, 2016

Published: March 23, 2016

\section{ABSTRACT}

Sensitive and non-invasive biomarkers for pancreatic cancer (PC) are lacking. We aimed to identify salivary long non-coding RNAs (IncRNAs) as biomarkers in diagnosis of resectable PC. Five well-documented IncRNAs: H19, HOTAIR, HOTTIP, MALAT1, PVT1, which are most closely associated with pancreatic cancer from previous studies were selected as putative IncRNA biomarkers. Their expression in pancreatic tissues and saliva of cancer patients and healthy controls was measured by quantification polymerase chain reaction (qPCR). Compared with benign pancreatic tumour (BPT) and normal pancreatic tissues (NPT), HOTAIR, HOTTIP and PVT1 were significantly upregulated in pancreatic cancer tissues (PCT). As compared to BPT or healthy groups, the salivary levels of HOTAIR and PVT1 were significantly higher in PC group. They were significantly reduced after the curative pancreatectomy. Both salivary IncRNAs distinguished PC patients from healthy controls and BPT patients with sensitivities and specificities ranging from $60-97 \%$. The expression of salivary HOTAIR and PVT1 did not differ significantly between healthy controls and any one of eight leading cancers worldwide. Collectively, our findings indicate that salivary HOTAIR and PVT1 show potential as novel non-invasive biomarkers for detecting PC.

\section{INTRODUCTION}

Pancreatic cancer (PC) has the poorest survival rate among all human cancer types. Its mortality is almost identical to incidence. The median survival time of PC is only 4 months [1]. Although, the 5 -year survival of postoperation for PC is only $20 \%$, surgical resection is the only potentially curative treatment. However, only $10-20 \%$ of patients are diagnosed with the disease at a resectable stage [2].

The pancreas is a small organ that is located deep within the body, which is part of the problem in detection via imaging modalities. More importantly, there are no specific symptoms for PC, and sensitive biomarkers are lacking. Hence, $\mathrm{PC}$ is often diagnosed at late stage. To date, carbohydrate antigen 19-9 (CA19-9) is the only biomarker related to PC for which U.S. Food and Drug Administration (FDA)-cleared diagnostics exist [3]. But serum CA19-9 has no role in screening asymptomatic populations, and shows a sensitivity and specificity of $79-81 \%$ and $82-90 \%$ respectively for the diagnosis of $\mathrm{PC}$ in symptomatic patients. Vital limitations to CA19-9 diagnosis in PC include poor sensitivity, false negative results in Lewis negative phenotypes of blood groups (5-10\%) and increased false positivity in patients with obstructive jaundice (10-60\%) [4].Thus, there is an urgent need to find a sensitive and noninvasive biomarker for screening early pancreatic cancer.

Long non-coding RNAs (lncRNAs) are transcripts longer than 200 nucleotides. IncRNAs activate several biological processes such as transcription, translation, gene expression etc. LncRNAs show great tissue specificity, making them attractive in the search of novel cancer biomarkers in body fluid samples. According to previous reports, lncRNAs: H19, HOTAIR (HOX transcript antisense intergenic RNA), HOTTIP (HOXA distal transcript antisense RNA), MALAT1 (metastasis-associated lung adenocarcinoma transcript 1), PVT1 (plasmacytoma variant 
translocation 1) are most closely associated with PC. There are all up-regulated in PC tissues or cell lines, exhibit pro-oncogenic activities and correlate with unfavorable prognosis. H19 promotes PC cell invasion and migration partially by enhancing HMGA2-mediated epithelialmesenchymal transition (EMT) via antagonizing let-7 [5]. Gene set enrichment analysis indicates that HOTAIR stimulates gene sets mainly associated with cell proliferation and cell cycle progression. HOTAIR knockdown in PC cells decreases cell proliferation, alters cell cycle progression, induces apoptosis and inhibits tumour growth in mouse xenograft model [6]. HOTTIP enhances cell proliferation, invasion, and chemoresistance by activating HOXA13 [7]. MALAT1 knowdown inhibits PC cell proliferation and reduces cell migration and invasion in vitro. The underlying mechanisms are possibly associated with inducing G2/M cell cycle arrest, promoting cell apoptosis, suppressing EMT and decreasing cancer stem-like properties [8]. Functional inactivation of the PVT1 contributes to enhanced Gemcitabine sensitivity in human pancreatic cancer ASPC-1 cells, vice versa [9].

Very limited studies report the diagnostic performance of circulating lncRNAs for detecting PC. Plasma fragments of HOTTIP-005 and RP11-567G11.1 were significantly upregulated in the PC patients. The sensitivity was $89 \%$ and $75.6 \%$ and specificity was $68.3 \%$ and $66.7 \%$, respectively, for discriminating PC from non-PC [10]. But blood collection is still invasive, and can cause pain and infection. A large blood supply flows into the salivary glands. Most compounds found in blood are also present in saliva, such as DNA, RNA, protein, drugs, and viruses. In the USA, commercial products for saliva-based diagnosis of drug abuse and HIV infection and for assessing hormone levels and various toxicology issues have been approved by the FDA and are in widespread use [11]. Studies report that salivary transcripts, proteins, metabolites, and other molecules may serve as biomarkers to detect oral cancer, breast cancer, lung cancer, ovarian cancer, esophageal cancer, Sjögren's syndrome, and other oral and systemic diseases $[12,13]$. Thus, saliva can be a desirable body fluid for biomarker detection in clinical applications. The functions of H19, HOTAIR, HOTTIP, MALAT1, and PVT1 in PC have been partly understood, but their salivary levels for detecting PC remained unclear. We aimed to explore whether they could serve as non-invasive biomarkers in saliva to detect resectable PC in this study.

\section{RESULTS}

\section{Basic participant information}

From 2011 to 2015,55 patients with resectable pancreatic cancer, 20 patients with benign pancreatic tumour were enrolled in this study. Their diagnosis was confirmed by pathology. 55 healthy individuals matched with PC patients in age, gender, and ethnicity were recuited. Their basic information is summarized in Table 1.

\section{The expression of the putative IncRNA biomarkers in tissue and saliva}

This study consisted of a discovery phase and a verification phase, followed by a validation phase. Five well-documented lncRNAs (H19, HOTAIR, HOTTIP, MALAT1, PVT1) most closely associated with PC from previous studies were selected as putative lncRNA biomarkers. Their expression levels of different kinds of pancreatic tissues were analyzed in the discovery phase to select candidate biomarkers. Potential biomarkers were established in the verification phase based on their differential salivary levels between the healthy controls and the patients. In validation phase, the specificities of the potential salivary biomarkers were further validated by comparing their expression between healthy controls and patients with any one of eight leading cancers worldwide (Figure 1).

Compared with normal pancreatic tissues (NPT), the expression of HOTAIR, HOTTIP, and PVT1 exhibited significant elevation in pancreatic cancer tissues (PCT) ( $p$ values were 0.025, 0.006, and 0.016, respectively). Meanwhile, H19 and MALAT1 levels did not significantly differ between the two groups ( $p$ values were 0.262 and 0.423 , respectively). And H19, HOTAIR, HOTTIP, MALAT1, and PVT1 levels did not significantly differ between NPT and benign pancreatic tumours (BPT) tissues ( $p$ values were 0.522 , $0.759,0.200,0.631$, and 1.000 , respectively). Compared with BPT, HOTAIR, HOTTIP, and PVT1 were also showed significantly higher expression levels in PCT ( $p$ values were $0.016,0.016$, and 0.006 , respectively). But H19 and MALAT1 showed no significant differences between the two groups, ( $p$ values were 0.337 and 0.423 , respectively) (Figure 2 ).

Compared with healthy controls, salivary HOTAIR and PVT1 showed significant over-expression in PC patients (both $p$ values were $<0.001$ ), but there were no significant differences of salivary H19, HOTTIP, and MALAT1 levels between the two groups ( $p$ values were $0.149,0.630$ and 0.639 , respectively). Salivary levels of H19, HOTAIR, HOTTIP, MALAT1 and PVT1 did not differ significantly between healthy and BPT groups ( $p$ values were $0.666,0.260,0.458,0.533$ and 0.060 , respectively). Additionally, significant differences were showed between salivary levels of HOTAIR and PVT1 in PC and BPT patients (both $p$ values were $<0.001$ ), but no significant differences were obtained in salivary H19, HOTTIP, and MALAT1 levels between the two groups ( $p$ values were $0.200,0.623,0.733$, respectively) (Figure 3 ).

\section{The expression alteration of salivary HOTAIR and PVT1 after the curative pancreatectomy}

In the randomly selected six PC patients receiving curative pancreatectomy, their salivary levels of 
Table 1: Demographic and clinical information of enrolled participants

\begin{tabular}{|c|c|c|c|c|c|}
\hline $\begin{array}{l}\text { Demographic } \\
\text { variables }\end{array}$ & Characteristics & $\begin{array}{l}\text { Pancreatic } \\
\text { cancer }(n=55)\end{array}$ & $\begin{array}{l}\text { Healthy control } \\
(n=55)\end{array}$ & $\begin{array}{l}\text { Benign } \\
\text { pancreatic } \\
\text { tumour }(n=20)\end{array}$ & $P$ Value* \\
\hline \multirow[t]{3}{*}{ Age (years) } & & & & & 0.141 \\
\hline & Mean \pm SD & $60.7 \pm 12.5$ & $57.1 \pm 11.3$ & $51.3 \pm 13.2$ & \\
\hline & Median (range) & $61(13-81)$ & $57(23-71)$ & $49.0(34-79)$ & \\
\hline \multirow[t]{3}{*}{ Sex } & & & & & 0.842 \\
\hline & Male & 36 & 35 & 14 & \\
\hline & Female & 19 & 20 & 6 & \\
\hline Ethnicity & Han Chinese & 55 & 55 & 20 & 1 \\
\hline \multicolumn{6}{|l|}{ CA19-9 } \\
\hline & $\geq 37 \mathrm{U} / \mathrm{mL}$ & 25 & 0 & 10 & \\
\hline & $<37 \mathrm{U} / \mathrm{mL}$ & 30 & 55 & 10 & \\
\hline \multicolumn{6}{|l|}{ Tumour position } \\
\hline & Head and neck & 42 & & 11 & \\
\hline & Body and tail & 13 & & 9 & \\
\hline \multicolumn{6}{|l|}{ Tumour size } \\
\hline & $\geq 4 \mathrm{~cm}$ & 30 & & 6 & \\
\hline & $<4 \mathrm{~cm}$ & 25 & & 14 & \\
\hline \multicolumn{6}{|l|}{ Cancer staging } \\
\hline & I & 9 & & & \\
\hline & II & 14 & & & \\
\hline & III & 32 & & & \\
\hline \multicolumn{6}{|l|}{$\begin{array}{l}\text { Histopathological } \\
\text { type }\end{array}$} \\
\hline & $\begin{array}{l}\text { Ductal adenocar- } \\
\text { cinoma }\end{array}$ & 49 & $\begin{array}{l}\text { Chronic inflam- } \\
\text { mation }\end{array}$ & 10 & \\
\hline & $\begin{array}{l}\text { Acinar cell carci- } \\
\text { noma }\end{array}$ & 3 & $\begin{array}{l}\text { Papillary epithe- } \\
\text { lioma }\end{array}$ & 4 & \\
\hline & $\begin{array}{l}\text { Mucinous cystad- } \\
\text { enocarcinoma }\end{array}$ & 3 & \#Others & 6 & \\
\hline
\end{tabular}

*The $P$-value represents the difference between pancreatic cancer patients and healthy controls. \#Other histopathological types include autoimmune pancreatitis $(n=2)$, mucinus cystadenoma $(n=1)$, fibroplasias $(n=1)$, leiomyoma $(n=1)$, granulomatous inflammation $(n=1)$. 


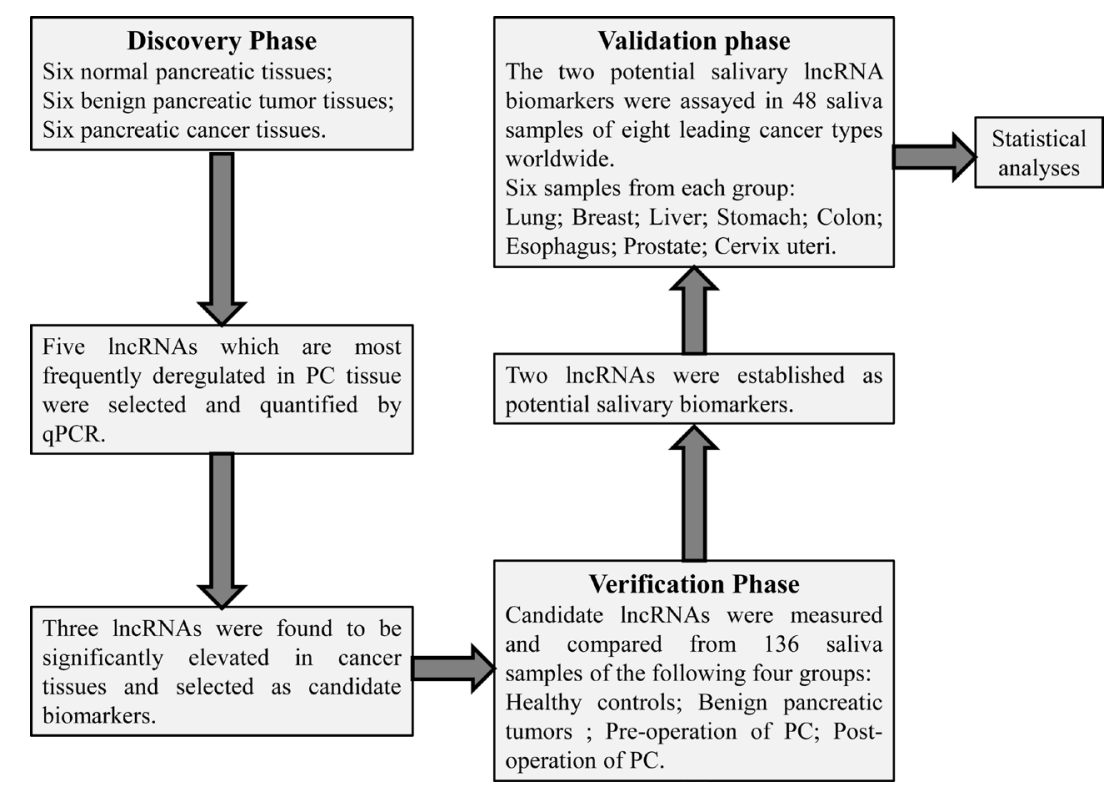

Figure 1: The flowchart of this study. Five well-documented lncRNAs (H19, HOTAIR, HOTTIP, MALAT1, PVT1) closely associated with pancreatic cancer from previous studies were selected as putative lncRNA biomarkers. Their expression levels were firstly quantified and compared within different types of pancreatic tissue in the discovery phase. In the verification phase, their expression in saliva was compared within healthy controls, benign pancreatic tumour, pre-operative samples of pancreatic cancer (PC), and post-operative samples of PC. HOTAIR and PVT1 were established as potential salivary lncRNA biomarkers, and their expressions were also analyzed in saliva of patients with any one of eight leading cancer types worldwide. Finally the discriminatory power of salivary HOTAIR and PVT1 was obtained by statistical analyses.

A

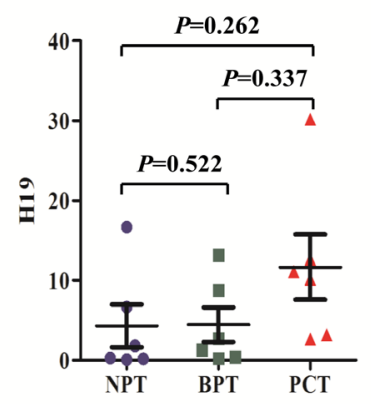

B

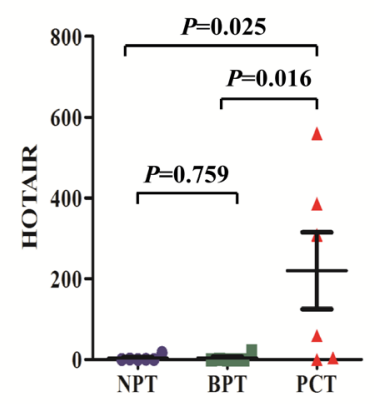

C

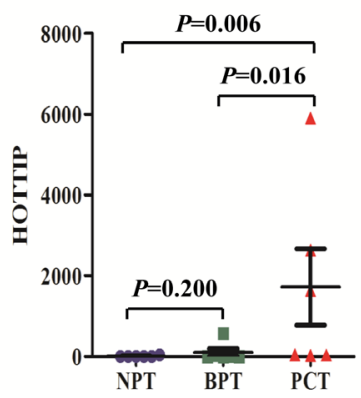

$\mathbf{E}$

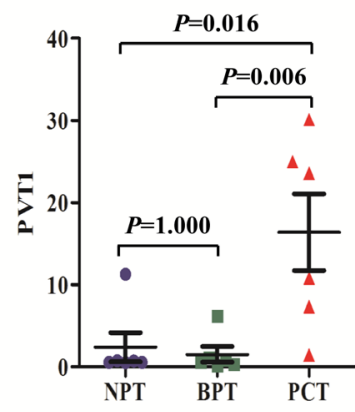

Figure 2: The expression of putative IncRNA biomarkers in pancreatic tissues. The expression of H19 (A) and MALAT1 (D) did not differ significantly between pancreatic cancer tissues (PCT) and normal pancreatic tissues (NPT) or benign pancreatic tumour (BPT) tissues. Meanwhile, as compared to NPT or BPT tissues, the expression of HOTAIR (B) HOTTIP (C) and PVT1 (E) exhibited significant elevation in PCT. There were no statistical differences in H19, HOTAIR, HOTTIP, MALAT1 and PVT1 levels between NPT and BPT tissues. The bars represent the standard errors, and the lines across the bars denote the means. 
HOTAIR and PVT1 were compared before and after the surgery. The six patients were followed up at least six months, and CT or/and MRI did not indicate cancer recurrence in them. The post-operative saliva samples of the patients were collected over six months after the surgery. Salivary HOTAIR and PVT1 levels were significantly reduced after surgery (both $p$ values were $<0.05$ ) (Figure 4). And their post-operative levels also returned to normal levels judging from no significant differences of their levels between the post-operative and healthy groups ( $P$ values were 0.790 and 0.287 , respectively).

\section{The discriminatory power of salivary HOTAIR and PVT1 to detect PC}

Receiver Operating Characteristic (ROC) curves were constructed and used to evaluate the discriminatory power of salivary HOTAIR and PVT1 for differentiating between two groups. An optimal cut-off value is needed for ROC curve to define the discriminatory power. When the Youden index (Youden index = sensitivity + specificity -1) reaches the maximum value, the corresponding cut-off value will yield the highest sum of sensitivity and specificity.

After calculation of the Youden index, if the cut-off value was determined to be 2.168921, salivary
HOTAIR differentiated PC from healthy control with sensitivity of $78.2 \%$, and specificity of $85.6 \%$ (Figure 5A). And it differentiated PC from BPT with sensitivity of $80.0 \%$, and specificity of $90.0 \%$ (Cut-off value $=1.98554)$ (Figure 5B). Additionally, salivary PVT1 yielded sensitivity of $96.4 \%$, and specificity of $63.6 \%$ for distinguishing PC from healthy controls (Cut-off value $=1.23991$ ) (Figure 5C). And it yielded sensitivity of $69.1 \%$, and specificity of $95.0 \%$ for distinguishing PC from BPT (Cut-off value $=3.11795$ ) (Figure 5D). Through logistic regression model, both of the discriminatory power of salivary HOTAIR and PVT1 were combined. Statistically, the area under the curve (AUC) is bigger, the discriminatory power is better. Thus, judging by the AUCs, the discriminatory power of the combination was improved. According to the logistic regression model, and supposing that the salivary levels of HOTAIR and PVT1 are X1 and X2, the combination formula was $\mathrm{F} 1=-4.183+1.716 \mathrm{X} 1+$ $0.265 X 2$. Accordingly salivary HOTAIR + PVT1 combination differentiated PC from healthy control with sensitivity of $78.2 \%$, and specificity of $90.9 \%$ (Cut-off value $=0.17256)($ Figure $5 \mathrm{E})$. And it differentiated PC from BPT with sensitivity of $81.8 \%$, and specificity of $95.0 \%$ (The combination formula F2 $=-3.917+1.66 \mathrm{X} 1$ $+0.59 \mathrm{X} 2$, and cut-off value $=0.770875)($ Figure $5 \mathrm{~F})$. The results are summarized in Table 2.
A

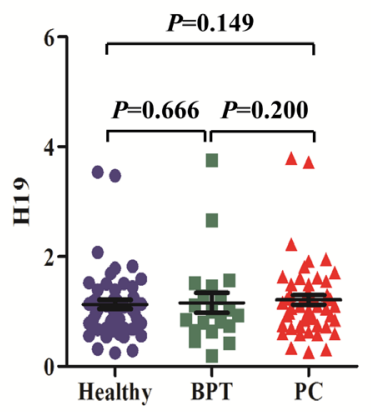

D

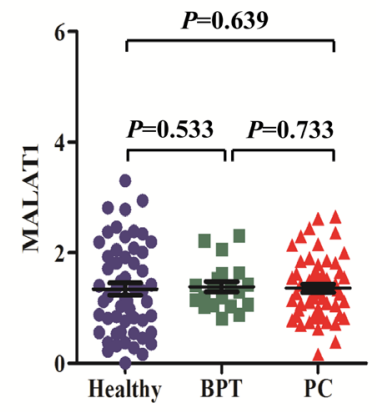

B

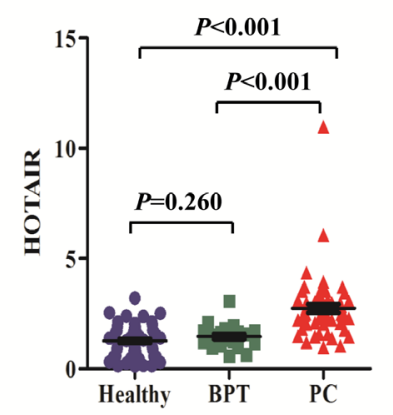

C

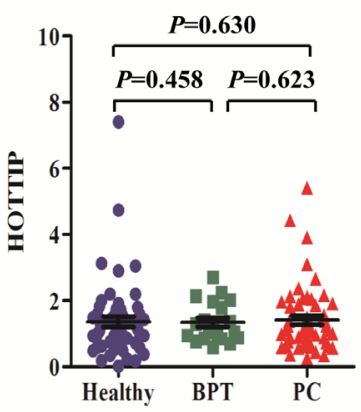

$\mathbf{E}$

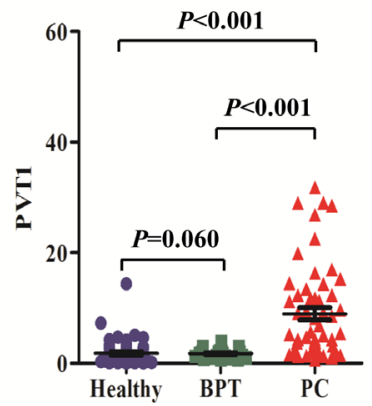

Figure 3: The expression of putative IncRNA biomarkers in saliva. There were no expression differences of salivary H19 (A) HOTTIP (C) and MALAT1 (D) between healthy, BPT, and PC groups. But salivary HOTAIR (B) and PVT1 (E) levels showed significant over-expression in PC group compared with healthy or BPT group. No significant difference was observed about salivary H19, HOTAIR, HOTTIP, MALAT1 and PVT1 levels between healthy and BPT groups. 
A

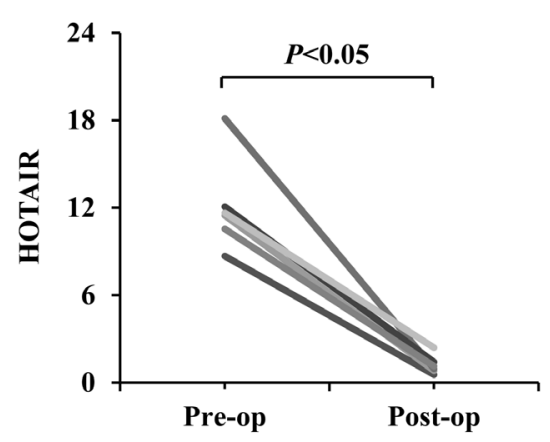

$\mathbf{C}$

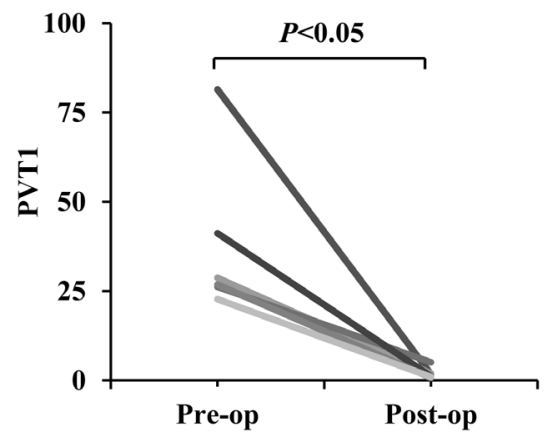

B

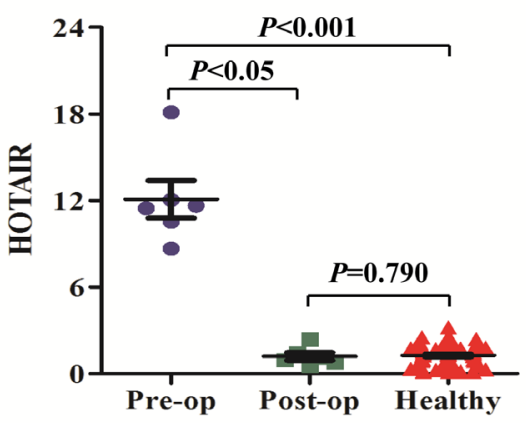

D

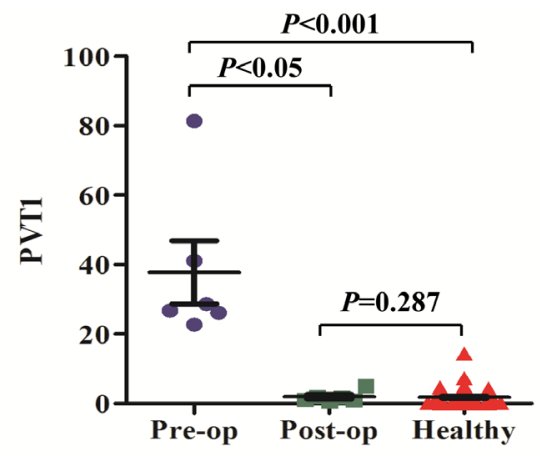

Figure 4: The expression alteration of salivary HOTAIR and PVT1 after the curative pancreatectomy. Salivary HOTAIR $(\mathbf{A}, \mathbf{B})$ and PVT1 (C, D) levels were significantly reduced in the post-operative (Post-op) samples when compared to the pre-operative (Pre-op) samples. No significant differences were obtained when their pre-operative levels were compared with the controls.

A

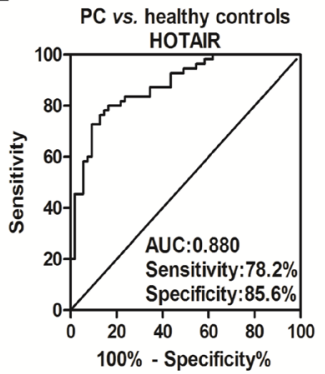

D

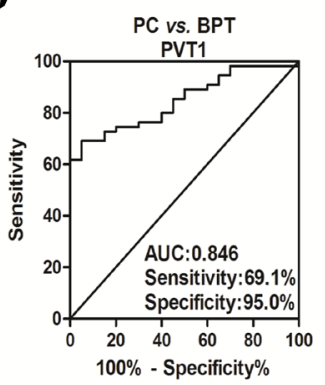

B

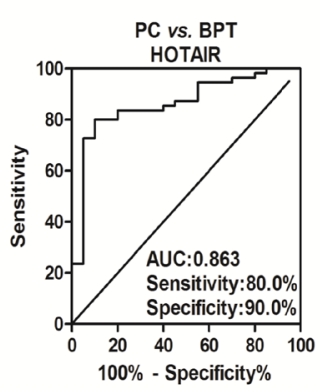

$\mathbf{E}$

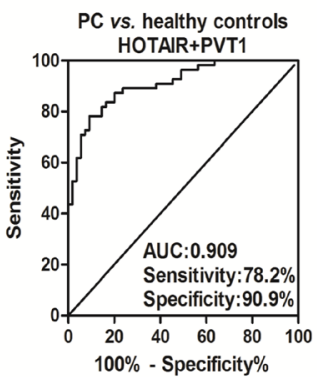

C

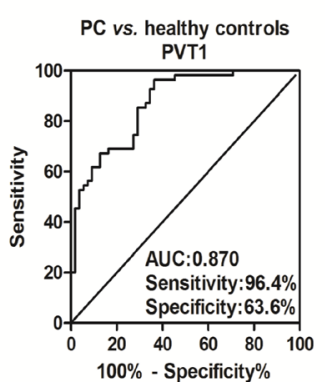

$\mathbf{F}$

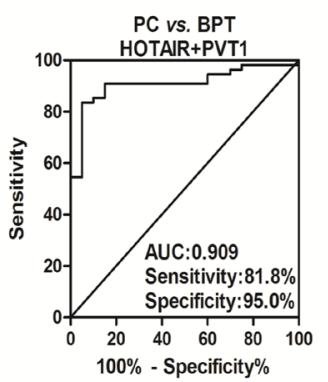

Figure 5: The ROC curves of salivary HOTAIR and PVT1 for detecting PC. Salivary HOTAIR differentiated PC from healthy control with sensitivity of $78.2 \%$, and specificity of $85.6 \%$ (A). And it differentiated PC from BPT with sensitivity of $80.0 \%$, and specificity of $90.0 \%$ (B). Salivary PVT1 yielded sensitivity of $96.4 \%$, and specificity of $63.6 \%$ for distinguishing PC from healthy controls (C). And it yielded sensitivity of $69.1 \%$, and specificity of $95.0 \%$ for distinguishing PC from BPT (D). The sensitivity of salivary HOTAIR + PVT1 combination was $78.2 \%$, and specificity was $90.9 \%$ for discriminating PC from healthy controls (E). And the combination discriminated PC from BPT with sensitivity of $81.8 \%$, and specificity of $95.0 \%(\mathbf{F})$. 
Table 2: The discriminatory power of salivary HOTAIR and PVT1 for the detection of resectable pancreatic cancer

PC vs. healthy control

PC vs. BPT

\begin{tabular}{|l|l|c|c|c|c|c|c|c|}
\hline LncRNA & $\boldsymbol{P}$ & AUC & Sensitivity & Specificity & $\boldsymbol{P}$ & AUC & Sensitivity & Specificity \\
\hline HOTAIR & $<0.001$ & 0.880 & $78.2 \%$, & $85.6 \%$ & $<0.001$ & 0.863 & $80.0 \%$ & $90.0 \%$ \\
PVT1 & $<0.001$ & 0.870 & $96.4 \%$ & $63.6 \%$ & $<0.001$ & 0.846 & $69.1 \%$ & $95.0 \%$ \\
HOTAIR + PVT1 & $<0.001$ & 0.909 & $78.2 \%$ & $90.9 \%$ & $<0.001$ & 0.909 & $81.8 \%$ & $95.0 \%$ \\
\hline
\end{tabular}

\section{The discriminatory power of salivary HOTAIR and PVT1 for detecting PC patients with CA19-9 $<37 \mathrm{U} / \mathrm{ml}$}

To date, CA19-9 is the only biomarker related to $\mathrm{PC}$ for which FDA-cleared diagnostics exist. We analyzed whether the potential lncRNA biomarkers in saliva could show better discriminatory power in detecting PC than that of serum CA19-9. According to the international criteria, the upper limit of serum CA19-9 for healthy people is 37 $\mathrm{U} / \mathrm{ml}$. In this study, 25 of 55 PC patients showed CA19-9 levels exceeding this limit, indicating that serum CA19-9 showed a sensitivity of $45.5 \%(25 / 55)$ for the detection of resectable PC (Figure 6A). Meanwhile, 10 of $20 \mathrm{BPT}$ patients exhibited CA19-9 levels > $37 \mathrm{U} / \mathrm{ml}$, implying that serum CA19-9 showed a specificity of 50\% (10/20) (Figure 6B) for discriminating patients with benign pancreatic tumours from malignant ones. Compared with healthy controls, the expression of salivary HOTAIR and PVT1 in both groups of PC with serum CA19-9 $<37 \mathrm{U} / \mathrm{ml}$ and CA19-9 $>37 \mathrm{U} / \mathrm{ml}$ showed significant up-regulation (all $P$ values were $<0.001$ ) (Figure 6C, 6D). But their expression levels were not significantly higher in both groups of BPT with serum CA19-9 $<37$ U/ml and CA19$9>37 \mathrm{U} / \mathrm{ml}$ (Figure 6E, 6F).

According to the ROC curve analysis, salivary HOTAIR discriminated PC with serum CA19-9 $<37 \mathrm{U} / \mathrm{ml}$ from healthy controls with sensitivity and specificity of $70.0 \%$ and $87.3 \%$, respectively (Cut-off value $=2.063118$ ) (Figure 7A). And salivary PVT1 discriminated PC with serum CA19-9 $<37 \mathrm{U} / \mathrm{ml}$ with sensitivity and specificity of $63.3 \%$ and $100 \%$, respectively (Cut-off value $=3.473288$ ) (Figure 7B). After combining their discriminatory power by the logistic regression model, their combined sensitivity and specificity for detecting PC with serum CA19-9 $<37 \mathrm{U} / \mathrm{ml}$ were $70.0 \%, 92.7 \%$, respectively (The combination formula F3 $=-3.965+0.627 \mathrm{X} 1+0.937 \mathrm{X} 2$, and cut-off value $=-0.13145)$ (Figure $7 \mathrm{C})$.

\section{The expression of the salivary HOTAIR and PVT1 in other leading cancers}

In order to preliminarily evaluate the specificities of the two potential lncRNA biomarkers, their expression levels were assessed in total 48 saliva samples of eight leading causes of cancer-related deaths worldwide, including lung, breast, liver, stomach, colon, esophagus, prostate, cervix uteri cancers (six samples from each cancer) [14], and compared with that of healthy controls. The expression of salivary HOTAIR and PVT1 did not differ significantly between any one of the aforementioned cancers and healthy controls (Figure 8).

\section{DISCUSSION}

To our knowledge, this is the first study to report salivary lncRNA biomarker for detecting human disease. According to the measurement by qPCR and statistical analyses, salivary HOTAIR and PVT1 were potentially useful biomarkers, with good discriminatory power for differentiating PC from healthy controls and BPT. The sensitivities and specificities ranged from 60-100\%. After the curative pancreatectomy without recurrence for over six months, the expression levels of salivary HOTAIR and PVT1 in the PC patients were significantly reduced and return to normal levels. This result primarily implied that the aberrant expression of HOTAIR and PVT1 was closely associated with or mainly derived from distal pancreatic tumour. Additionally, this pilot study preliminarily found that the levels of salivary HOTAIR and PVT1 in patients with any one of the eight leading cancers worldwide did not differ significantly with that of healthy controls. And the PC patients in this study were recruited based on no concurrent oral, systemic and infectious diseases. Thus, although the sample sizes of other leading cancers were small, this result implies that salivary HOTAIR and PVT1 show potential to be a novel and specific biomarker for PC. But studies with larger sample sizes and other less common cancers are still needed to prove their specificities to PC. Finally, the salivary levels of the two lncRNAs did not differ significantly between BPT and healthy groups, indicating that the two lncRNAs could be deregulated in saliva only when PC developed.

The most common serum biomarker used for PC is CA19-9, which is over-expressed in pancreatic and other gastrointestinal tumours, including esophageal and gastric cancers. Additionally, it cannot distinguish between cancer and chronic pancreatitis and possibly 
other disease states with chronic inflammation [15]. In this study, less than one half of PC patients and one half of BPT patients showed elevated levels of serum CA19-9. Salivary HOTAIR and PVT1 were not significantly elevated in BPT patients and patients with any one of eight leading cancers worldwide. Hence, in terms of the non-invasiveness and convenience, and better diagnostic performance, salivary HOTAIR and PVT1 might take the place of CA19-9 for PC detection in the future. But these findings still need large-scale validation. Statistically, when the discriminatory power

A

$$
\text { PC patients }(N=55)
$$

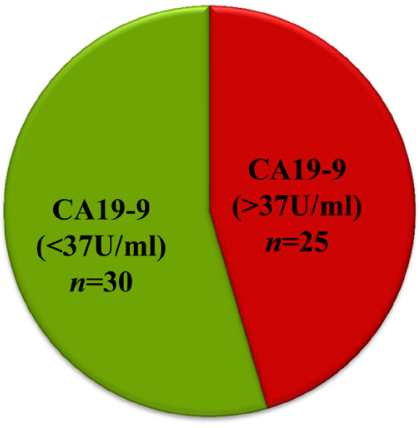

C

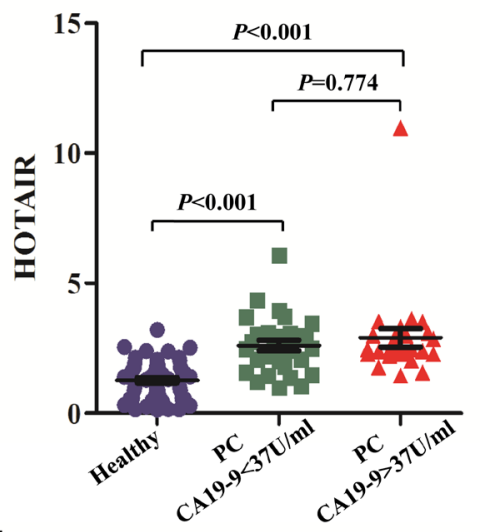

E

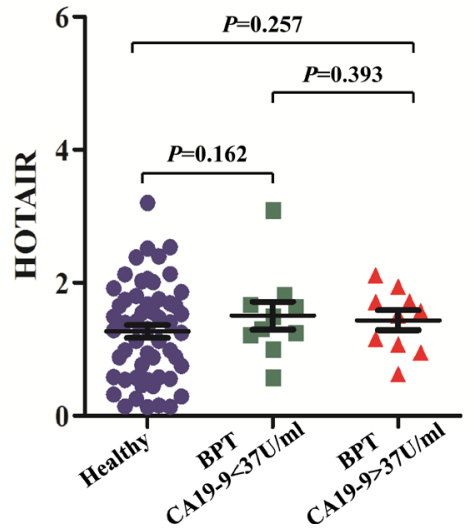

of each biomarker was combined via logistic regression model, the combined discriminatory power would be improved judging by the AUCs. Interestingly, when the discriminatory power of salivary HOTAIR and PVT1 for detecting PC with serum CA19-9 $<37 \mathrm{U} / \mathrm{ml}$ was combined, the combined AUC did not increase. The reason might be that both salivary levels of HOTAIR and PVT1 were normal in some PC patients with serum CA19-9 $<37 \mathrm{U} / \mathrm{ml}$. Thus, both makers cannot make up for each other to detect PC with serum CA19-9 $<37 \mathrm{U} / \mathrm{ml}$. Although mRNAs have been considered to be fragile and

B

BPT patients $(N=20)$

D
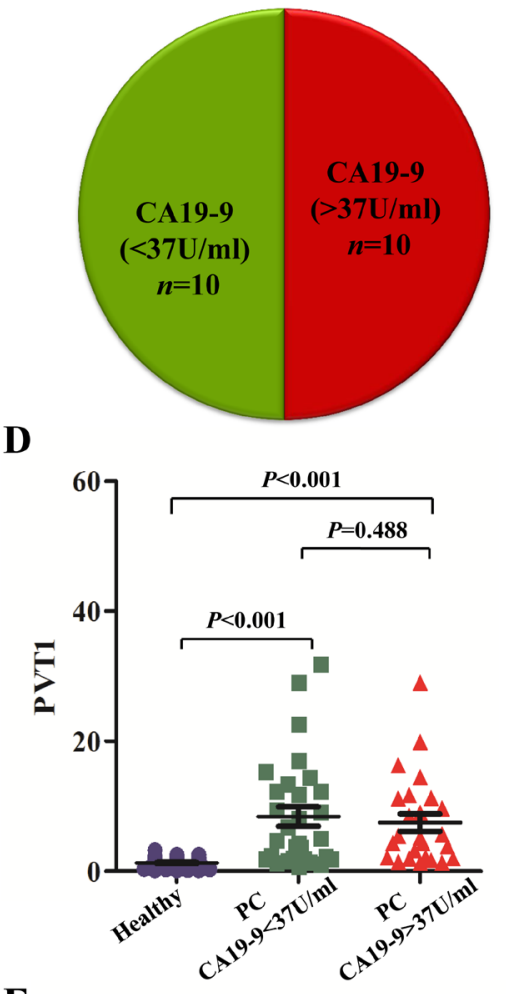

$\mathbf{F}$

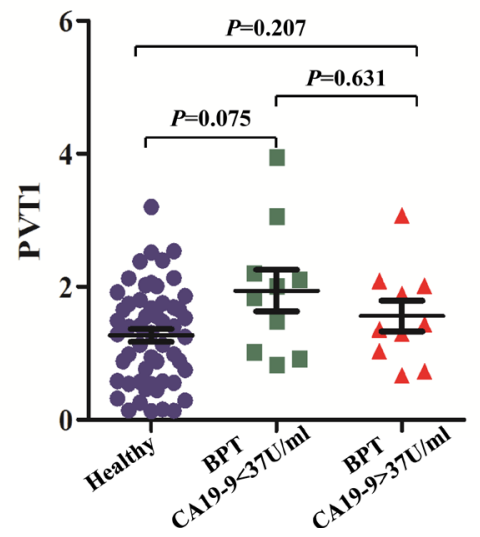

Figure 6: The aberrant expression of salivary HOTAIR and PVT1 in PC with different CA19-9 ranges. In this study, 25 of 55 (45.5\%) PC patients showed CA19-9 levels exceeding the normal limit of $<37 \mathrm{U} / \mathrm{ml}$ (A). Meanwhile, 10 of 20 (50\%) BPT patients exhibited CA19-9 > $37 \mathrm{U} / \mathrm{ml}$ (B). Compared with healthy controls, the expression of salivary HOTAIR (C) and PVT1 (D) in both groups of PC with serum CA19-9 $<37 \mathrm{U} / \mathrm{ml}$ and $>37 \mathrm{U} / \mathrm{ml}$ showed significant up-regulation, but their expression levels were not significantly deregulated in both groups of BPT with serum CA19-9 $<37 \mathrm{U} / \mathrm{ml}$ and $>37 \mathrm{U} / \mathrm{ml}(\mathbf{E}, \mathbf{F})$. 
A

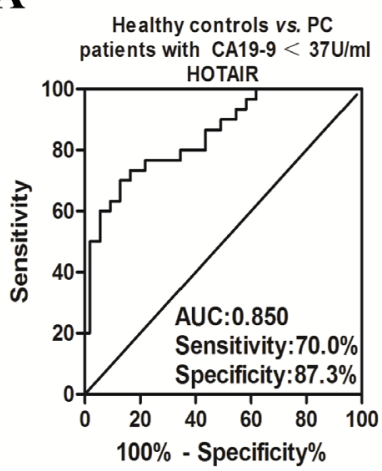

B

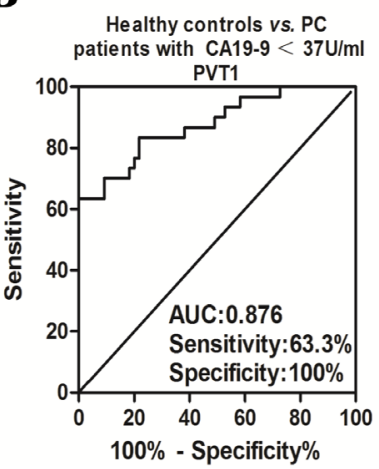

$\mathbf{C}$

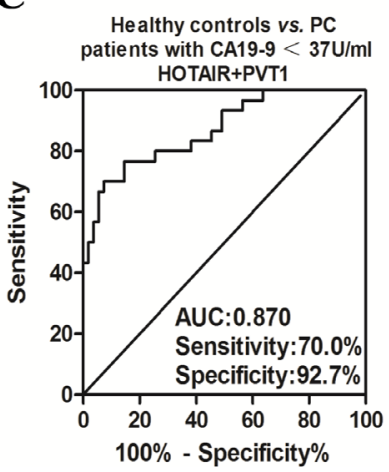

Figure 7: The ROC curves of salivary HOTAIR and PVT1 for detecting PC with different CA19-9 ranges. Salivary HOTAIR discriminated PC with serum CA19-9 $<37 \mathrm{U} / \mathrm{ml}$ from healthy controls with sensitivity and specificity of $70.0 \%$ and $87.3 \%$, respectively (A). And salivary PVT1 showed sensitivity and specificity of $63.3 \%$ and $100 \%$ in discriminating PC with serum CA19-9 $<37 \mathrm{U} / \mathrm{ml}$ from healthy controls (B). After combining their discriminatory power, their combined sensitivity and specificity for detecting PC with serum CA19-9 $<37 \mathrm{U} / \mathrm{ml}$ were $70.0 \%, 92.7 \%$, respectively $(\mathbf{C})$.
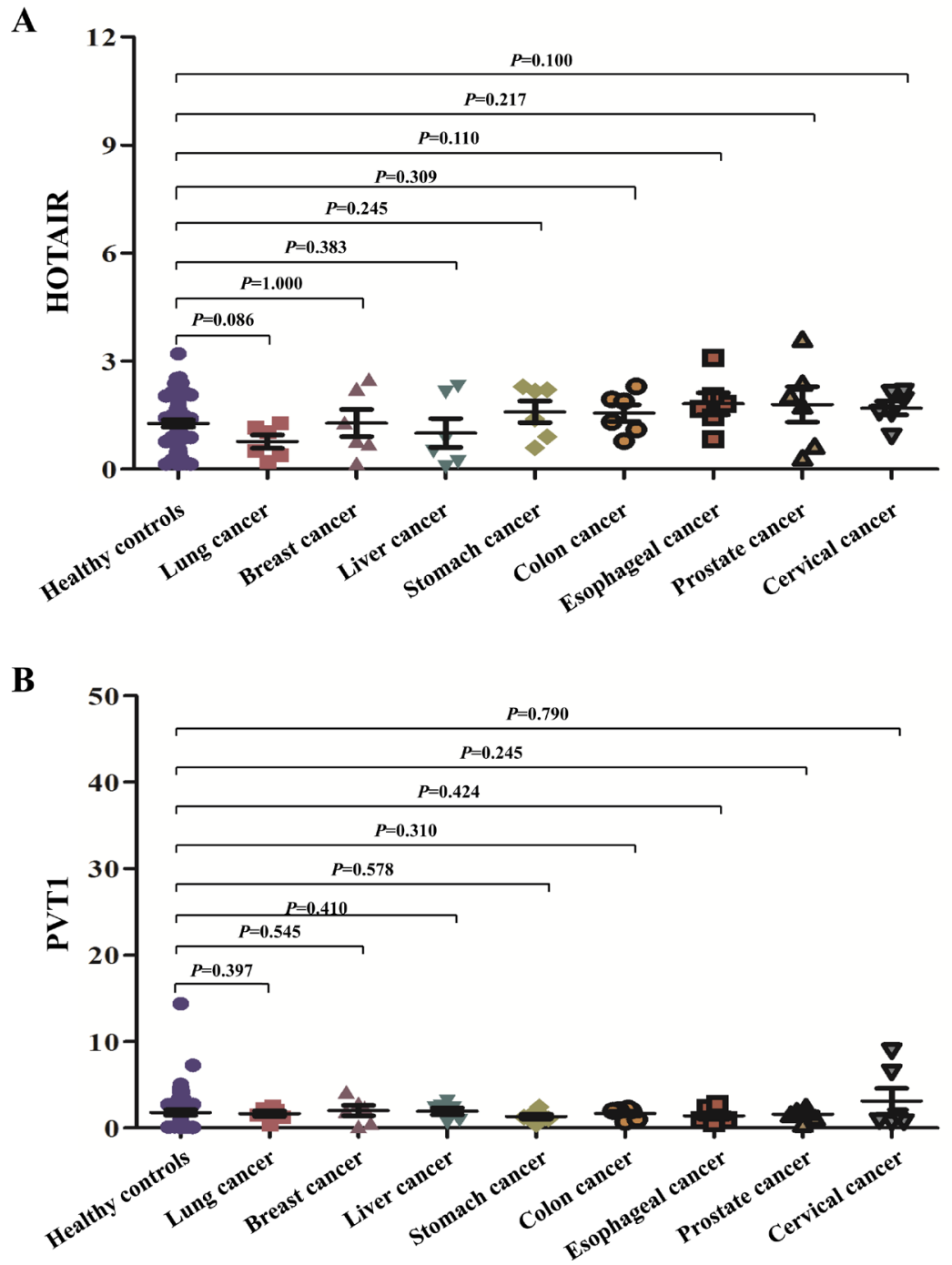

Figure 8: The expression of the salivary HOTAIR and PVT1 in other leading cancers. The expression of salivary HOTAIR (A) and PVT1 (B) did not differ significantly between healthy controls and any one of eight leading cancers worldwide. 
easily degrade, lncRNAs were found to be more stable. Harsh environment, i.e. multiple freeze-thaw cycles, strong acid, base and RNase treatment had hardly any effect on the levels of lncRNAs [16]. Thus, lncRNAs can be readily detected by qPCR from the body fluids, i.e. serum, plasma, gastric liquids, or urine [17]. But blood and gastric liquid collection is invasive. Urine collection is not applicable for anuretic patients. Saliva collection is non-invasive, simple, low-cost, practical and comfortable compared with other invasive methods. Thus, saliva is an ideal body fluid for biomarker detection in clinical applications. It is unclear whether the cancerspecific RNAs detected in the circulation originate from tumour cell death and lysis or whether they are actively secreted by tumour cells or blood cells. Although dozens of molecular biomarkers in saliva were identified for detecting different diseases, the rationale and relationship between systemic diseases and saliva biomarkers are unclear. Gao et al. [18] developed a panel of salivary transcriptomic biomarkers discriminatory for melanoma and lung cancer in the tumour-bearing rodent model. They speculate that systemic networks are present in our body, which allows communication between distal organs and the salivary glands. Signals transmitted through such networks can activate related signaling pathways that lead to altered gene expression and thereby produce diseaseinduced salivary biomarker profiles. Such disease-induced salivary gland-mediated transcriptomes can serve as valuable biomarker of disease onset and/or progression. Therefore, the salivary glands can be regarded as a reactive organ monitoring systemic diseases and saliva can be investigated as a biomarker-enriched disease-reflective biofluid. Zhang et al. [19] hypothesize that tumours produce mediators (hormones/cytokines/lymphokines) which can regulate the activities and gene expression patterns of distal organs (salivary glands) through the circulation. Upon contact with the salivary gland, cancerspecific mediators elicit altered gene expression profiles and transcription of associated RNAs that are secreted into the saliva. Both HOTAIR and PVT1 were elevated in PC tissue and saliva of PC patients. So they may be mainly derived from PC cells. HOTTIP was increased in PC tissue but not in saliva of PC patients in this study. According to aforementioned theories, we reason that through the circulation, PC-produced mediators contact the salivary glands, and subsequently elicit altered expression of HOTAIR and PVT1 genes but not HOTTIP gene.

Limitations exist in this study. First, microarray was not conducted to make lncRNA profiles of PC. So other potential lncRNA biomarkers may also exist. Second the sample size was small, and the subjects were limited to a Chinese Han population. Multi-centre studies with larger sample sizes are needed to further validate our findings. Third, the biological functions of HOTAIR and PVT1 were based on previous reports. In-depth analysis of their biological functions is needed. Fourth, saliva samples of patients with recurrent PC after the pancreatectomy were not collected. Thus, salivary levels of HOTAIR and PVT1 after PC recurrence need further investigation in the future.

In conclusion, differential expression of salivary HOTAIR and PVT1 may closely correlate with distal pancreatic cancer, and they can be novel non-invasive biomarkers with good sensitivity and specificity, better than serum CA19-9 for detecting PC. This study provides a new method and prospect for the early detection of pancreatic cancer or other systemic diseases using a noninvasive screening method.

\section{MATERIALS AND METHODS}

\section{Subject selection}

From 2011 to 2015, 18 tissue samples consisted of six samples of PC tissue, six tissues of benign pancreatic tumour (BPT), and six normal pancreatic tissues (NPT) resected from adjacent BPT. Saliva was collected as previous described [20, 21]. Saliva samples from 55 pre-operative patients with resectable PC, and 20 with BPT were collected. Six saliva samples from postoperative PC patients over six months after the curative panreatectomy were also procured. At time of the postoperative sample procurement, no cancer recurrence was implicated in the CT or MRI examinations of the six patients. Total 48 saliva samples of eight leading cancer types worldwide (six samples from each cancer) [14]: lung, breast, liver, stomach, colon, esophagus, prostate, and cervix uteri cancers (six samples from each group) were also obtained. All patient histopathology results were confirmed by pathology, and no concurrent oral and other systemic diseases were diagnosed, such as gingivitis, tuberculosis, systemic lupus erythematosus et al. Patients with a diagnosis of other malignancies and receiving chemotherapy and radiotherapy prior to saliva collection were not recruited neither. Pancreatic cancers were either resectable or borderline resectable according to National Comprehensive Cancer Network: Practice Guidelines in Oncology v.2.2010 [22]. Details are presented in Supplementary Material 1. Cancer staging was based on the American Joint Committee on Cancer: the 7th Edition of the AJCC Cancer Staging [23]. Fiftyfive saliva samples from healthy individuals matched with PC patients in age, gender, and ethnicity were obtained. Institutional review boards or ethics committees at all participating institutes approved the study protocol. All participants were provided written consent for their information to be stored in the hospital database. Brief demographic information of all patients is presented in Supplementary Material 2. 


\section{Protocols for the detection of IncRNAs}

Total RNAs were extracted from frozen tissues and $1.2 \mathrm{ml}$ of saliva using TRIzol or the mirVana PARIS Kit, respectively (both from Life Technologies, USA) according to the manufacturer's protocols. The reverse transcription reaction of IncRNAs was performed with the ReverTra Ace qPCR RT Kit (Toyobo, Japan) and the cDNA solution was amplified using Maxima SYBR Green qPCR master mixes (Thermo Fisher Scientific, USA) following the manufacturer's protocols. qPCR reactions were run using an ABI 7500 Real-Time PCR System (Life Technologies, USA), and the reaction mixtures were incubated at $95^{\circ} \mathrm{C}$ for $10 \mathrm{~min}$, followed by 45 cycles of $95^{\circ} \mathrm{C}$ or $15 \mathrm{~s}$ and $60^{\circ} \mathrm{C}$ for $32 \mathrm{~s}$. Melt curve analysis was performed to validate the generation of the expected PCR products. Each sample was analyzed in triplicate. The expression levels of each lncRNA were normalized to that of $\beta$-actin. All expression levels were calculated using the $2^{-\Delta \Delta \mathrm{Ct}}$ method.

\section{Study design}

This study consisted of a discovery phase and a verification phase, followed by a validation phase. Five well-documented lncRNAs (H19, HOTAIR, HOTTIP, MALAT1, PVT1) most closely associated with PC from previous studies were selected as putative lncRNA biomarkers. In the discovery phase, the expression levels of the selected lncRNAs were measured by qPCR and compared within PCT, BPT tissues and NPT.

In the verification phase, the five lncRNAs were also subjected to validation by $\mathrm{qPCR}$ using a saliva sample set containing 55 patients with PC, 20 with BPT and 55 healthy controls. HOTAIR and PVT1 exhibiting statistically significance when comparing PC group with healthy or BPT group were identified as potential salivary biomarkers to detect PC.

In the validation phase, in order to preliminarily evaluate the specificities of the two potential salivary biomarkers, their expression levels were assessed in total 48 saliva samples of eight leading causes of cancer-related deaths worldwide, including lung, breast, liver, stomach, colon, esophagus, prostate, cervix uteri cancers, and compared with that of healthy controls.

\section{Statistical analysis}

LncRNA expression levels and ages were compared using the Mann Whitney $U$ test. Genders were compared using the $\chi^{2}$ test. The differences of IncRNAs before and after surgery were studied by Wilcoxon signed-rank test. ROC curves were used to evaluate the discriminatory power of each IncRNA for differentiating between two groups. The validated salivary biomarkers were constructed into binary logistic regression models, and step-wise backward model selection was performed to determine the final combinations of biomarkers. Statistical analyses were performed using the SPSS software (ver. 13.0). A two-tailed $P$ value $<0.05$ was considered statistically significant.

\section{FUNDING SUPPORTS}

The project was supported partly by grants from the National Key Basic Research Program of China (973 Program, 2012CB526700), the National Natural Science Foundation of China (U1501224, 81370511), and the Specialized Research Fund for the Doctoral Program of Higher Education (20120171110081).

\section{CONFLICTS OF INTEREST}

There is no conflict interest.

\section{Authors' contributions}

ZJX, XLC and JZL performed the experiments; YWG, HJL, XMP, JJ and HLL provided technical and material support; ZJX, XLC, JZL and BW analyzed data and drafted the paper; ZJX and BW designed and wrote the paper; BW supervised study.

\section{REFERENCES}

1. Sharma C, Eltawil KM, Renfrew PD, Walsh MJ, Molinari M. Advances in diagnosis, treatment and palliation of pancreatic carcinoma: 1990-2010. World J Gastroenterol. 2011; 17:867-897.

2. Poruk KE, Firpo MA, Adler DG, Mulvihill SJ. Screening for pancreatic cancer: Why, how, and who? Ann Surg. 2013; 257:17-26.

3. Wu E, Zhou S, Bhat K, Ma Q. CA 19-9 and pancreatic cancer. Clin Adv Hematol Oncol. 2013; 11:53-55.

4. Ballehaninna UK, Chamberlain RS. The clinical utility of serum CA 19-9 in the diagnosis, prognosis and management of pancreatic adenocarcinoma: An evidence based appraisal. J Gastrointest Oncol. 2012; 3:105-119.

5. Ma C, Nong K, Zhu H, Wang W, Huang X, Yuan Z, Ai K. H19 promotes pancreatic cancer metastasis by derepressing let-7's suppression on its target HMGA2-mediated EMT. Tumour Biol. 2014; 35:9163-9169.

6. Kim K, Jutooru I, Chadalapaka G, Johnson G, Frank J, Burghardt R, Kim S, Safe S. HOTAIR is a negative prognostic factor and exhibits pro-oncogenic activity in pancreatic cancer. Oncogene. 2013; 32:1616-1625.

7. Li Z, Zhao X, Zhou Y, Liu Y, Zhou Q, Ye H, Wang Y, Zeng J, Song Y, Gao W, Zheng S, Zhuang B, Chen H, et al. The long non-coding RNA HOTTIP promotes progression and gemcitabine resistance by regulating HOXA13 in pancreatic cancer. J Transl Med. 2015; 13:84. 
8. Jiao F, Hu H, Yuan C, Wang L, Jiang W, Jin Z, Guo Z, Wang L. Elevated expression level of long noncoding RNA MALAT-1 facilitates cell growth, migration and invasion in pancreatic cancer. Oncol Rep. 2014; 32:2485-2492.

9. You L, Chang D, Du HZ, Zhao YP. Genome-wide screen identifies PVT1 as a regulator of Gemcitabine sensitivity in human pancreatic cancer cells. Biochem Biophys Res Commun. 2011; 407:1-6.

10. Wang Y, Li Z, Zheng S, Zhou Y, Zhao L, Ye H, Zhao X, Gao W, Fu Z, Zhou Q, Liu Y, Chen R. Expression profile of long non-coding RNAs in pancreatic cancer and their clinical significance as biomarkers. Oncotarget. 2015; 6: 35684-35698. doi: 10.18632/oncotarget.5533.

11. Lee YH, Wong DT. Saliva: An emerging biofluid for early detection of diseases. Am J Dent. 2009; 22:241-248.

12. Zhang Y, Sun J, Lin CC, Abemayor E, Wang MB, Wong DT. The emerging landscape of salivary diagnostics. Oral Health Dent Manag. 2014; 13:200-210.

13. Xie ZJ, Chen G, Zhang XC, Li DF, Huang J, Li ZJ. Saliva supernatant miR-21: A novel potential biomarker for esophageal cancer detection. Asian Pac J Cancer Prev. 2012; 13:6145-6149.

14. Torre LA, Bray F, Siegel RL, Ferlay J, Lortet-Tieulent J, Jemal A. Global cancer statistics, 2012. CA Cancer J Clin. 2015; 65:87-108.

15. De La Cruz MS, Young AP, Ruffin MT. Diagnosis and management of pancreatic cancer. Am Fam Physician. 2014; 89:626-632.

16. Tong YS, Wang XW, Zhou XL, Liu ZH, Yang TX, Shi WH, Xie HW, Lv J, Wu QQ, Cao XF. Identification of the long non-coding RNA POU3F3 in plasma as a novel biomarker for diagnosis of esophageal squamous cell carcinoma. Mol Cancer. 2015; 14:3.
17. Kladi-Skandali A, Michaelidou K, Scorilas A, Mavridis K. Long noncoding RNAs in digestive system malignancies: A novel class of cancer biomarkers and therapeutic targets? Gastroenterol Res Pract. 2015; 2015:319861.

18. Gao K, Zhou H, Zhang L, Lee JW, Zhou Q, Hu S, Wolinsky LE, Farrell J, Eibl G, Wong DT. Systemic disease-induced salivary biomarker profiles in mouse models of melanoma and non-small cell lung cancer. PLoS One. 2009; 4:e5875.

19. Zhang L, Farrell JJ, Zhou H, Elashoff D, Akin D, Park NH, Chia D, Wong DT. Salivary transcriptomic biomarkers for detection of resectable pancreatic cancer. Gastroenterology. 2010; 138:949-957.

20. Xie Z, Chen G, Zhang X, Li D, Huang J, Yang C, Zhang P, Qin Y, Duan Y, Gong B, Li Z. Salivary microRNAs as promising biomarkers for detection of esophageal cancer. PLoS One. 2013; 8:e57502.

21. Xie Z, Yin X, Gong B, Nie W, Wu B, Zhang X, Huang J, Zhang P, Zhou Z, Li Z. Salivary microRNAs show potential as a noninvasive biomarker for detecting resectable pancreatic cancer. Cancer Prev Res (Phila). 2015; 8: 165-173.

22. Margaret AT, Joseph H, Martha BP, Pablo AJ, John PH, Aaron RS, Stephen B, Srinadh K, Sarah PT, Edgar BJ, Albert K, Robert SW, Boris $\mathrm{K}$, et al. National Comprehensive Cancer Network: Practice Guidelines in Oncology v.2.2010. Fort Washington, PA: National Comprehensive Cancer Network. 2011.

23. Edge S, Byrd DR, Compton CC, Fritz AG, Greene FL, Trotti A. AJCC cancer staging manual (7th ed). New York, NY:Springer. 2010. 